\title{
The Application of Approximation Characteristics Non-Linear Resistor Implement the Required Current-Voltage Characteristics
}

\author{
Vitaly Viktorovich Pivnev*, Sergey Nikolaevich Basan, \\ Yuri Petrovich Voloshchenko \\ 2a, Komsomolsk descent st., Taganrog, Rostov region, Russia \\ 2, Calarasi st., Tuapse, Krasnodar, Russia \\ 4b, Frunze st., Taganrog, Rostov region, Russia \\ natali_vit@list.ru, sbasan@mail.ru, yvoloshchenko@yandex.ru
}

\begin{abstract}
Keywords: Synthesis schemes, non-linear circuits, equivalent transformations, analog-digital-analog element, non-linear resistance, controllable voltage source, Taylor series, Maclaurin, Lagrange polynomials.

Abstract. In this paper, we consider the problem of development of equivalent circuit of non-linear element with the current-voltage characteristic controlled either voltage or current, which can be modeled without any changes of scheme in accordance with design requirements. For solving the problem we use an analog-digital-analog element. The current-voltage characteristic of this element is determined by the program of the digital device work. To approximate the characteristics of non-linear elements we apply polynomials of varying degrees - segments of the Taylor series, Maclaurin, Lagrange, et al. In our opinion, the solution of such a problem is relevant.
\end{abstract}

\section{Introduction}

Successes of a modern microelectronics allow at higher level a synthesize devices for solving tasks of simulation, automatic control, communication, and etc.

Majority of physical processes are non-linear. In this paper we consider only those processes that can be described in the relevant non-linear algebraic and nonlinear integral - differential equations. We assume that there are electrical circuits, currents and voltages of which up to indications equal to the corresponding symbols of physical quantities. Thus, the obtained model represents a non-linear electrical circuit that generally consists of linear and non-linear parts, which can be represented as shown in fig. 1.

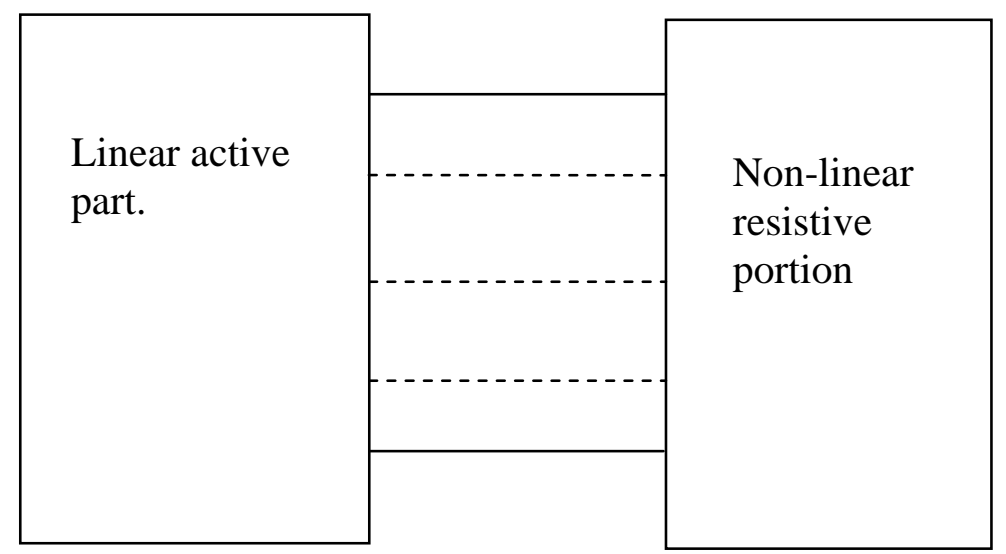

Fig.1. Non-linear electric circuit.

We assume also that the non-linear part consists only of two-pole elements, and the current-voltage (volt-ampere) characteristics of each element are known. The main problem is the implementation of two-poles with specified current-voltage characteristics is the large variety of features and technical difficulties in achieving accuracy and stability. These disadvantages are 
inherent to all known methods [1 - 7]. As a result, usually in the implementation of devices comprising of non-linear elements we need to perform adjustment.

The aim of this research is to develop a nonlinear equivalent circuit of a two-pole with a current-voltage characteristic of the controlled either voltage or current, which can be modeled without changing of the schema, in accordance with design requirements. In our opinion, the solution to this problem is topical.

Our solution is based on the use of analog-digital-analog element [5]. This element is a controllable voltage source (fig. 2).

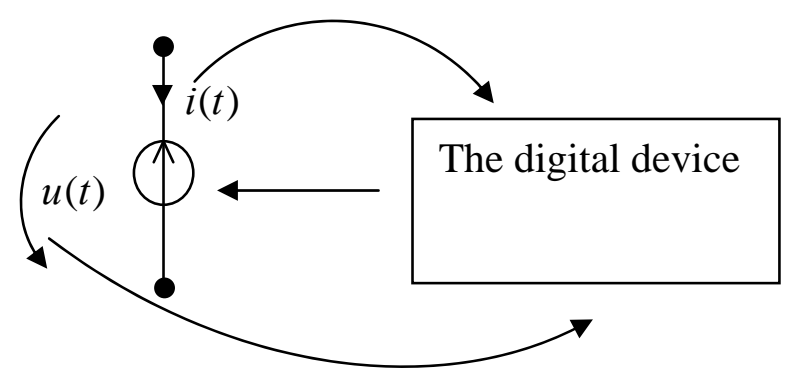

Fig.2. The analog-digital-analog element.

The current-voltage characteristic of this element is determined by of the digital device program. Thus, without changing the structure of the device in fig. 2, using only software, it is possible to obtain elements with different current-voltage characteristics.

Currently, to approximate the characteristics of nonlinear elements are widely used polynomials of varying degrees (segments of the Taylor series, Maclaurin, Lagrange polynomials, etc.) [1, 2, 7]. The pole equation for the equivalent circuit of the nonlinear element, has the form of a polynomial and shown in fig. 3.

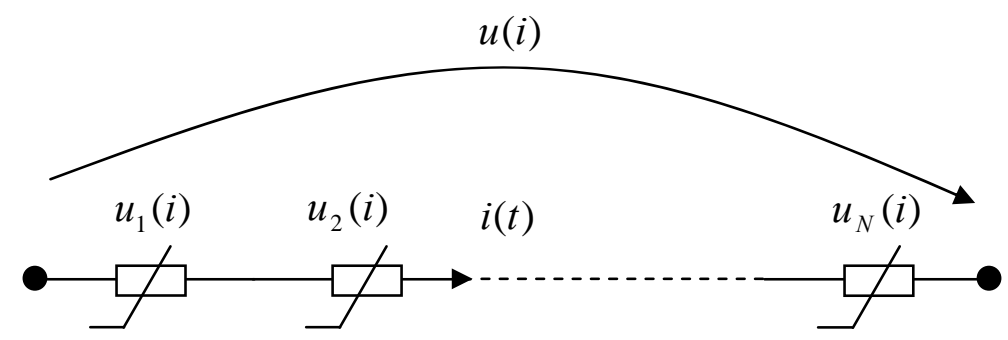

Fig.3. The equivalent circuit of the non-linear element, the equation has the form of a polynomial.

When you select the method of pole approximation equation, k-th element has the form as shown in fig. 3:

$$
u_{k}(i)=A_{k} i^{B_{k}}
$$

In this equation, $A_{k}, B_{k}$ - real numbers, $k=1 \div N$.

We synthesize the scheme of each element in fig.3 by scheme shown in fig.2. The expression (1) can be regarded as the solution of a differential equation. In this case, the numbers $A_{k}, B_{k}$ can be regarded as a constant of integration. Since in the expression (1) two permanent number (two constant of integration), then the desired differential equation is a differential equation of second order. Let's differentiate the equation (1) twice. 


$$
\begin{aligned}
& u_{k}^{\prime}=A_{k} B_{k} i^{\left(B_{k}-1\right)} \\
& u_{k}^{\prime \prime}=A_{k} B_{k}\left(B_{k}-1\right) i^{\left(B_{k}-2\right)} .
\end{aligned}
$$

After considering equation (1), (2) and (3) as a system of equations, we exclude the constants of integration from the equation (2).

$$
B_{k}=\frac{u_{k}^{\prime} i}{u_{k}} .
$$

If we divide the (3) (2), taking into account (4), we will obtain following:

$$
u_{k}^{\prime \prime}-\frac{\left(u_{k}^{\prime}\right)^{2}}{u_{k}}+\frac{u_{k}^{\prime}}{i}=0 .
$$

The resulting equation is the pole equation k-th element in the circuit in Fig. 3, which recorded in the differential form.

In order to integration constants satisfy a condition (1), it is necessary to provide the initial conditions that satisfy the equations (6) and (7):

$$
\begin{aligned}
& u_{H}=A i_{H}^{B}, \\
& u_{H}^{\prime}=B \frac{u_{H}}{i_{H}} .
\end{aligned}
$$

In cases where one of the coefficients A and B should not vary depending on the initial conditions, we can use the first order differential equation in simulation. For example, let's the coefficient B does not have to change depending on the initial conditions. Then in the expression (1) we consider only constant integration coefficient A.

From (1) it follows:

$$
A_{k}=\frac{u_{k}}{i^{B}}
$$

Differentiating (1) according to current, we obtain:

$$
u_{k}^{\prime}=A B i^{(B-1)} .
$$

Taking into account the expression (8) the ratio (9) can be converted to the form:

$$
u_{k}^{\prime}-B \frac{u_{k}}{i}=0
$$

The constant of integration in solving of the equation (10) could be found from the initial conditions:

$$
A=\frac{u_{H}}{i_{H}{ }^{B}}
$$


In this paper, we can make the following conclusions:

1. In the simulation of non-linear current-voltage characteristics of polynomials, equivalent circuit of each non-linear element can be represented as a series connection of the respective analog-digital-analog two-terminal devices.

2. Equation for analog-digital-analog two-pole devices can be written in differential form (5) or (10).

3. Expressions (5) or (10) are the basis for compiling of the algorithm of the digital devices(fig. 2).

4. In general, the mathematical equation describing the current-voltage nonlinear resistance characteristic may comprise a plurality of constant coefficients (e.g., n). Then the equation of this element is the differential equation of the $\mathrm{N}$-th order.

5. The presence in the digital part of the analog-digital-analog element allows you to perform debugging of electronic equipment by means of software and abandon the selection of parameters of analog components.

\section{Acknowledgements}

This work was supported by Russia's Southern Federal University grant: "Theory and methods of energy-saving control for distributed generation, transportation and electricity consumption systems".

\section{References}

[1] E. Filippov, Nonlinear Electrical Engineering, Energy, Moscov , 1986, 504 p.

[2] L.V.Danilov, P.N. Mathanov, E.S.Filippov, The theory of non-linear electric circuits, Energoatomizdat, Leningrad, 1990, 252 p.

[3] L.V. Danilov, Electrical circuit with non-linear R-element, Communications, Moscov, 1974, 135 p.

[4] S.N. Basan, Electrical circuits with nonlinear resistors, Taganrog Radio Engineering Institute named after V.D. Kalmikova, Rostov University Publishing, (1984), 200 p.

[5] S.N. Bazan, M.V. Izotov: Universal analog-digital electronic element equipment : Proceedings of the International Conference on "Radiation and scattering of electromagnetic wave IREMV-2009." - Taganrog, Izd Tsure, 2009. p. 486-489.

[6] Pivnev V.V., Basan S.N. Some questions about equivalent circuit synthesis and nonlinear electrical circuit implementation with the specified properties in the electronic simulation tasks: Applied Mechanics and Materials Vols. 670-671 (2014) P.1454-1457. - Access: http://www.scientific.net/AMM.670-671. 1454.

[7] Pivnev V.V., Basan S.N. Some the application of the Taylor series for the analysis of processes in non-linear resistive circuits : Applied Mechanics and Materials Vols. 701-702 (2015) P.1173-1176. - Access: http://www.scientific.net/AMM.701-702.1173. 